\title{
GeV-scale neutrinos at DUNE
}

\author{
Josu Hernandez-Garcia ${ }^{a *}$ \\ ${ }^{a}$ Institute for Theoretical Physics, ELTE Eötvös Loránd University \\ Pázmány Péter sétány 1/A, H-1117 Budapest, Hungary \\ E-mail: josu.hernandez@ttk.elte.hu
}

The simplest extension to account for neutrino masses requires the addition of heavy right-handed neutrinos. If these heavy neutrinos have masses up to the $\mathrm{GeV}$ scale, they could be produced via mixing at beam-dump experiments in leptonic and semileptonic meson decays. These long lived particles could eventually arrive and decay in the volume of the detector. In this poster, the capability of the DUNE near detector to look for these heavy neutral leptons is explored. First, the effective operators describing interactions of light mesons and one heavy neutrino will be reviewed. This effective Lagrangian is implemented in a FeynRules model file which is made publicly available so that fully differential event distributions can be simulated. Then, by computing the flux of heavy neutrinos at the near detector, we show that DUNE will be able to explore large regions of the allowed parameter space which could explain neutrino masses, through a type-I Seesaw.

\footnotetext{
*** The European Physical Society Conference on High Energy Physics (EPS-HEP2021), ***

*** 26-30 July $2021 * * *$

*** Online conference, jointly organized by Universität Hamburg and the research center DESY ***
}

\footnotetext{
${ }^{*}$ Speaker
} 


\section{Introduction}

One of the most important open windows to look for New Physics is the neutrino sector. The overwhelming neutrino oscillation phenomena implies that neutrinos are massive and that lepton flavor is violated. The simplest extension of the Standard Model (SM) to accommodate neutrino masses consists in the addition of heavy right-handed neutrinos that are singlets of the SM gauge group [1-4]. The are known as heavy neutral leptons (HNLs). At least two HNLs are needed in order to explain the observed atmospheric and solar neutrino mass splittings.

Then the flavor neutrinos $v_{\alpha}$, produced via charged currents interactions from the corresponding charged lepton $\ell_{\alpha}$, will thus correspond to a combination of the light $v_{i}$, and heavy $N_{i}$ states

$$
v_{\alpha}=\sum_{i=1}^{3} U_{\alpha i} v_{i}+\sum_{i=4}^{3+n} U_{\alpha i} N_{i},
$$

where $U_{\alpha i}$ is the unitary mixing matrix that diagonalizes the neutrino masses. If only one of these HNLs is light enough, or has a sufficiently large mixing to be produced in the experiment, all the phenomenology will be described by 3 mixing elements $U_{\alpha 4}$ and its mass $M_{4}$. Depending on the mass range of these HNLs, we can search for signatures in different experiments. In this project, we focus on the mass range from $10 \mathrm{MeV}$ to few $\mathrm{GeV}$, where peak searches at beam-dump experiments become a powerful tool to test this SM extension. In particular, the capabilities of the proposed Deep Underground Neutrino Experiment (DUNE) near detector (ND) as a beam-dump experiment to probe for these HNLs will be analyzed [5].

\section{The DUNE Near Detector as a beam-dump experiment}

The $120 \mathrm{GeV}$ protons from the Main Injector are dumped into a target producing light mesons $\left(\pi, K, D, D_{s}\right)$ which decay inside the decay pipe producing a neutrino beam. Thus, HNLs are produced in the decay pipe via their mixing with the active neutrinos. Depending on the energy and on the trajectory of the HNLs, they will propagate and eventually decay via mixing inside the volume of the ND giving, for example, a characteristic signal of two electrons and a neutrino. The ND complex will be located $574 \mathrm{~m}$ downstream from the neutrino beam source, where a highpressure gaseous TPC, a cylinder of $5 \mathrm{~m}$ diameter and $5 \mathrm{~m}$ length, surrounded by an electromagnetic calorimeter (ECAL) in a 0.5 T magnetic field, called the Multi-Purpose Detector (MPD) is located (see the DUNE Technical Design Report [6]). We will consider the detector volume corresponding to the MPD for which the beam-induced background is smaller given its lower density. See Figure 1 for an schematic layout of the HNL production and decay at DUNE ND.

The total number of expected HNL decay events inside the ND, for a given channel $c$ goes like

$$
N_{c}(\mathrm{ND})=\mathrm{BR}_{c} \times \int d E_{N} e^{-\frac{\Gamma L}{\gamma \beta}}\left(1-e^{-\frac{\Gamma \Delta \ell}{\gamma \beta}}\right) \frac{d \phi_{N}}{d E_{N}},
$$

where $\Gamma$ is the total decay width of the heavy neutrino in its rest frame, $L$ is the distance between the HNL production and the ND while $\Delta \ell$ is the length of the HNL trajectory inside the detector. Finally, for our sensitivity contours we estimate the $90 \%$ confidence level (CL) sensitivity on the signal following the Feldman and Cousins [7] prescription for a Poisson distribution with no background and under the hypothesis of no events being observed, which corresponds to the expected number of signal events being smaller than 2.44 . 


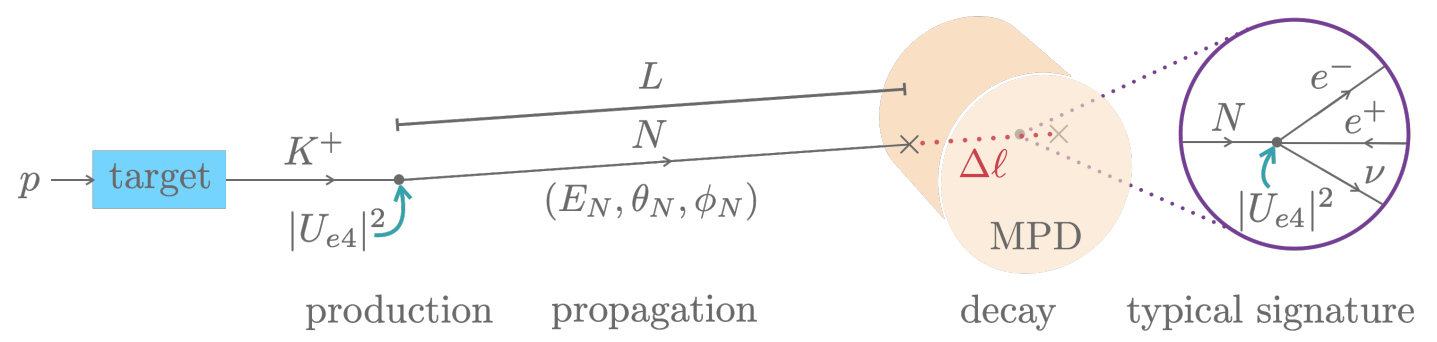

Figure 1: Schematic layout of the DUNE beam, target, decay pipe and MPD near detector.

\section{Effective interactions of light mesons and HNLs}

By integrating out the vector bosons, and replacing the corresponding hadronic matrix element, the effective operators describing interactions between light mesons (up to $2 \mathrm{GeV}$ ) and HNLs.

\subsection{Pseudoscalar interactions}

The hadronic matrix element between the vacuum and the pseudoscalar meson $P$ goes like

$$
\left\langle 0\left|j_{a \mu}^{A}\right| P_{b}\right\rangle=i \delta_{a b} \frac{f_{P}}{\sqrt{2}} p_{\mu},
$$

with $j_{a \mu}^{A}$, the corresponding axial current, and where $f_{P}$ and $p_{\mu}$ stand for the decay constant and the momentum of the pseudoscalar meson, respectively. Assuming on-shell particles, the effective operators describing the interaction between a pseudoscalar meson and a pair of leptons, can be written as a function of the fermion masses. For instance, the corresponding operators for the charged and neutral pion read

$$
\begin{aligned}
O_{\pi \ell_{\alpha} \bar{n}_{i}} & =i \sqrt{2} G_{F} U_{\alpha i} V_{u d} f_{\pi} \bar{\ell}_{\alpha}\left(m_{\alpha} P_{L}-m_{i} P_{R}\right) n_{i} \pi^{-}+\text {h.c. } \\
O_{\pi^{0} n_{i} \bar{n}_{j}} & =\frac{i}{2} G_{F} \sum_{\alpha} U_{\alpha i}^{\dagger} U_{\alpha j} f_{\pi} \bar{n}_{i}\left(m_{i} P_{L}-m_{j} P_{R}\right) n_{j} \pi^{0}+\text { h.c. }
\end{aligned}
$$

where we see that the decay will be dominated by the heavy states (in this case the HNL), in complete analogy to the chiral enhancement of the $\pi \rightarrow \mu v$ over $\pi \rightarrow e v$ decay.

\subsection{Vector interactions}

On the other hand, the hadronic matrix element of the vector mesons $V$ goes with the polarization vector $\epsilon_{\mu}$ instead

$$
\left\langle 0\left|j_{a \mu}^{V}\right| V_{b}\right\rangle=\delta_{a b} \frac{f_{V}}{\sqrt{2}} \epsilon_{\mu} .
$$

In a similar way, the operators describing vector meson interactions (for instance the one of rho meson) with HNLs are given by

$$
\begin{aligned}
O_{\rho \ell_{\alpha} \bar{n}_{i}} & =-\sqrt{2} G_{F} U_{\alpha i} V_{u d} f_{\rho} \rho_{\mu}^{-}\left(\bar{\ell}_{\alpha} \gamma^{\mu} P_{L} n_{i}\right)+\text { h.c. } \\
O_{\rho^{0} n_{i} \bar{n}_{j}} & =-\frac{1}{2} G_{F} \sum_{\alpha} U_{\alpha i}^{\dagger} U_{\alpha j}\left(1-2 s_{w}^{2}\right) f_{\rho} \rho_{\mu}^{0}\left(\bar{n}_{i} \gamma^{\mu} P_{L} n_{j}\right)+\text { h.c. }
\end{aligned}
$$




\subsection{Semileptonic meson decays}

Finally, we include the decay of a heavy pseudoscalar $P$ into a lighter one $D$, a charged lepton, and a neutrino (such as $K \rightarrow \pi \ell n$ ). In this case, the hadronic matrix element is usually given in terms of the two form factors $f_{+}$and $f_{-}[8,9]$ as a function of the momentum $q$ transferred between the two mesons

$$
\begin{aligned}
O_{P D \ell_{\alpha} \bar{n}_{i}=} & \sqrt{2} G_{F} V_{q q^{\prime}} U_{\alpha i} \bar{\ell}_{\alpha}\left[\left(f_{+}\left(q^{2}\right)-f_{-}\left(q^{2}\right)\right)\left(m_{\alpha} P_{L}-m_{i} P_{R}\right) \phi_{D}\right. \\
& \left.-2 i f_{+}\left(q^{2}\right)\left(\partial_{\mu} \phi_{D}\right) \gamma^{\mu} P_{L}\right] n_{i} \phi_{P}^{\dagger}+\text { h.c. }
\end{aligned}
$$

These effective op. have been implemented in a FeynRules [10] model file, publicly available here [11]. The UFO generated from the model file can be interplayed with MadGraph [12] for easy simulation of fully differential HNL production and decay events, scanning over the free parameters.

\section{Results and Conclusions}

The expected sensitivity of the proposed MPD of DUNE at 90\% CL for 7 years of data taking and assuming negligible background for $20 \%$ signal efficiency cuts [13] has been computed. The three panels of Figure 2 show the bounds on the plane $\left|U_{\alpha 4}\right|^{2}-M_{4}$, assuming that the HNL is coupled only to $e, \mu$, and $\tau$, respectively. The blue line corresponds to the expected bound for the DUNE ND derived from this work, while the shaded gray areas indicate the parameter space already excluded by direct searches in current experiments (see Ref [5] for the complete list of experiments). We find that DUNE is expected to improve over present constraints by several orders of magnitude in a large fraction of the parameter space and, in particular, for HNL masses between the $K$ and $D$ meson thresholds.

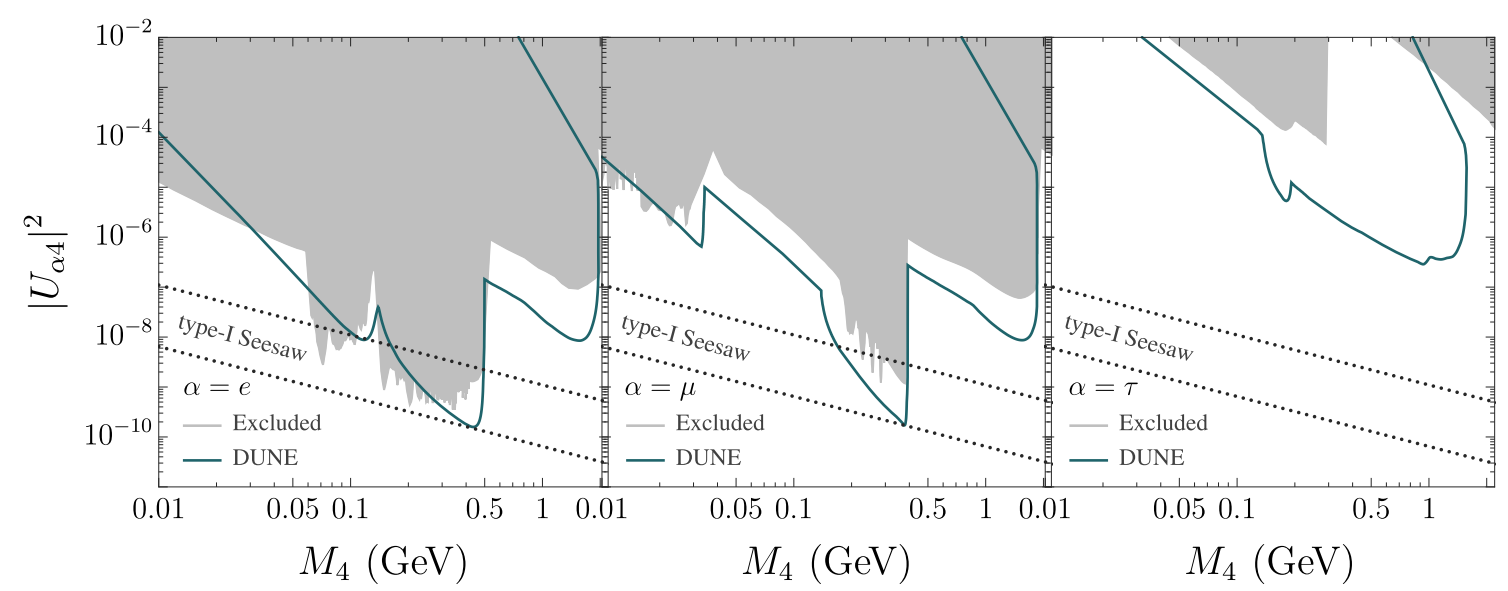

Figure 2: Expected DUNE sensitivity (at $90 \% \mathrm{CL}$ ) to the mixing matrix elements $\left|U_{\alpha 4}\right|^{2}$ as a function of the heavy neutrino mass. See text for details.

The dotted lines in Figure 2 represent the naive expectation for the mixing matrix elements from the Seesaw mechanism: $\left|U_{\alpha 4}\right|^{2} \sim m_{i} / M_{4}$, where $m_{i}$ stands for the light neutrino masses. We find that DUNE will be able to start exploring this region, for HNL masses close to the $K$ mass. 


\section{References}

[1] P. Minkowski, $\mu \rightarrow$ ey at a Rate of One Out of $10^{9}$ Muon Decays?, Phys. Lett. 67B (1977) 421-428.

[2] R. N. Mohapatra and G. Senjanovic, Neutrino Mass and Spontaneous Parity Nonconservation, Phys. Rev. Lett. 44 (1980) 912.

[3] T. Yanagida, Horizontal gauge symmetry and masses of neutrinos, Conf. Proc. C7902131 (1979) 95-99.

[4] M. Gell-Mann, P. Ramond, and R. Slansky, Complex Spinors and Unified Theories, Conf. Proc. C790927 (1979) 315-321, arXiv: 1306.4669 [hep-th].

[5] P. Coloma, E. Fernández-Martínez, M. González-López, J. Hernández-García and Z. Pavlovic, Eur. Phys. J. C 81 (2021) no.1, 78 [arXiv:2007.03701 [hep-ph]].

[6] DUNE Collaboration, B. Abi et al., Deep Underground Neutrino Experiment (DUNE), Far Detector Technical Design Report, Volume I Introduction to DUNE, arXiv:2002.02967 [physics.ins-det].

[7] G. J. Feldman and R. D. Cousins, A Unified approach to the classical statistical analysis of small signals, Phys. Rev. D 57 (1998) 3873-3889, arXiv: physics/9711021.

[8] ETM Collaboration, V. Lubicz, L. Riggio, G. Salerno, S. Simula, and C. Tarantino, Scalar and vector form factors of $D \rightarrow \pi(K) \ell v$ decays with $N_{f}=2+1+1$ twisted fermions, Phys. Rev. D96 (2017) 054514, arXiv:1706.03017 [hep-lat]. [erratum: Phys. Rev.D99, 099902(2019)].

[9] J. Bijnens, G. Colangelo, G. Ecker, and J. Gasser, Semileptonic kaon decays, 2nd DAPHNE Physics Handbook: 315-389 (1994) arXiv: hep-ph/9411311 [hep-ph].

[10] A. Alloul, N. D. Christensen, C. Degrande, C. Duhr, and B. Fuks, FeynRules 2.0 - A complete toolbox for tree-level phenomenology, Comput. Phys. Commun. 185 (2014) 2250-2300, arXiv:1310.1921 [hep-ph].

[11] https://feynrules.irmp.ucl.ac.be/wiki/HNLs.

[12] J. Alwall, R. Frederix, S. Frixione, V. Hirschi, F. Maltoni, O. Mattelaer, H. S. Shao, T. Stelzer, P. Torrielli, and M. Zaro, The automated computation of tree-level and next-to-leading order differential cross sections, and their matching to parton shower simulations, JHEP 07 (2014) 079, arXiv: 1405.0301 [hep-ph].

[13] T2K Collaboration, K. Abe et al., Search for heavy neutrinos with the T2K near detector ND280, Phys. Rev. D100 (2019) 052006, arXiv: 1902.07598 [hep-ex].

[14] KATRIN Collaboration, M. Aker et al., Improved Upper Limit on the Neutrino Mass from a Direct Kinematic Method by KATRIN, Phys. Rev. Lett. 123 (2019) 221802, arXiv: 1909.06048 [hep-ex]. 